\title{
Basic Biology of Mesenchymal Stem Cells
}

\author{
Karen Bieback \\ Institute of Transfusion Medicine and Immunology, Medical Faculty Mannheim, University of Heidelberg, Germany
}

A population of stem cells variously named mesenchymal stem cells, marrow stromal cells or more recently, in an attempt at standardization, mesenchymal stromal cells, but all luckily abbreviated MSC, has emerged as a highly interesting candidate for cellular therapies [1]. These cells have been known for a number of years to be key regulators of hematopoiesis within the bone marrow stroma [2]. They came into focus as regenerative therapeutics as they have been shown to differentiate along mesodermal lineages and to highly varying degrees possibly also to neuronal, hepatic and cardiac phenotypes [3]. The initial hope of an embryonal-like differentiation potential of MSC has not been fully realized as more recent data indicate that in only few cases MSC differentiation leads to therapeutic benefits but rather paracrine factors attract endogeneous repair mechanisms [4]. Cellular regeneration as a major therapeutic branch has been overtaken by immunomodulation. MSC exhibit impressive immunomodulatory capacities, which led to their use in the management of treatment-resistant graft-versus-host disease [5]. Additionally, MSC are under intense scrutiny in various clinical trials ranging from orthopedic approaches to treatment of graft-versus-host disease (www.clinicaltrials.gov). Despite their use in numerous clinical trials, the basic biology of these cells remains mainly ambiguous, cumulating in a complete lack of standardization. However, one should assume that understanding the basic biological characteristics should be the prerequisite for extensive clinical use. This issue of TRANSFUSION MEDICINE AND HEMOTHERAPY summarizes recent advances in understanding the basic biological properties of MSC.

The review article of Schäfer and Northoff [6] preludes the topic of MSC. Summarizing the basic parameters classically used for basal characterization as well as current knowledge about MSC, from issues of nomenclature to MSC basic characterization, the authors introduce open questions, such as 'Are MSC true stem cells?'. They also allude to the fact that safety issues have not been addressed adequately so far since, although controversially discussed, some authors report on malignant transformation of expanded MSC and others on facilitated tumor growth after transplantation.

Bourin et al. [7] delve into the aspect of tissue origin, isolation, and expansion protocols. Despite bone marrow remaining the major source for obtaining MSC, almost any tissue investigated has been shown to contain MSC. The authors provide an overview on different sources ranging from bone marrow to adipose tissue and different fetal sources. In general, MSC are generated by a variety of protocols, further hampering progress in standardization. Accordingly, the authors highlight several parameters which should be taken into account, ranging from cell densities to passage numbers and culture conditions including oxygen tension, media supplements as well as growth factor effects.

Focusing on the heterogeneous nature of MSC preparations, Rojewski, et al. [8] provide a comprehensive review of markers used to characterize but also to prospectively isolate MSC. Surface protein expression assessed by flow cytometry has emerged as valuable tool to define stem cell populations. However, MSC characterization by means of surface marker expression has been hampered by the fact that no single marker molecule is known that can discriminate MSC from other contaminating cells. In addition, various approaches aim to define pluripotent subpopulations of MSC, but again intrinsic as well as assay-specific factors complicate comparing results. Markers allowing for a prospective isolation are highly desirable, but although different proteins have been tried none of them showed reproducible results.

Highlighting aspects of isolation, expansion and phenotypic characterization, the subsequent articles focus on the functional characterization. The first from Wagner et al. [9] summarizes recent knowledge on the cell-cell interaction between MSC and hematopoietic stem cells. As known for years, the very close interaction between bone marrow stroma and hematopoietic stem cells, known as bone marrow stem cell niche,

\begin{tabular}{ll}
\hline KARGER & $\oplus$ 2008 S. Karger GmbH, Freiburg \\
Fax +49 7614520714 & Accessible online at: \\
$\begin{array}{l}\text { E-mail Information@Karger.de } \\
\text { www.karger.com }\end{array}$ & www.karger.com/tmh
\end{tabular}

Karen Bieback, Ph.D.

Institute of Transfusion Medicine and Immunology

German Red Cross Blood Service of Baden-Württemberg - Hessen

Medical Faculty Mannheim, University of Heidelberg

Friedrich-Ebert-Straße 107, 68167 Mannheim, Germany

Tel. +49 621 3706-8216; Fax -851, E-mail karen.bieback@medma.uni-heidelberg.de 
regulates self-renewal and differentiation of the latter. Attempts to replace classical bone marrow stroma with MSC to establish an in vitro model of the stem cell niche and to finally expand hematopoietic stem cells are reviewed. Influences of growth factors as well as other soluble mediators, oxygen tension, extracellular matrix but also cell-cell contact molecules are described as mechanisms constituting the stem cell niche.

Not only interactions with hematopoietic stem cells, but also their mature immunological functional progeny has received focus since the initial observations that MSC have significant immunomodulatory capacities. As highlighted in the article by Bifari et al. [10], MSC do not only play a role in hematopoiesis and especially in lymphopoiesis but rather exert regulatory activity on effector cells of both the innate and adaptive immune system. At present, no unique mechanism responsible for MSC immune regulation has been identified, but associated mechanisms are alluded to within this review.

As the differentiation potential has been the major attribute of MSC paving their way to regenerative medicine, recent advances in understanding the molecular control of differentiation have provided insights into MSC biology. The work of Gimble et al. [11] highlights studies on the in vitro differentiation potential of mainly two MSC populations derived from either bone marrow or adipose tissue. As further specified within the subsequent article of Quinn and Flake [12], in vitro differentiation assays of course inherit numerous limitations. Nevertheless they provide a deep insight into the underlying mechanisms including signaling pathways and transcriptional regulation. Frith and Genever [13] discuss the transcriptional control of differentiation events in MSC. They focus on key factors consistently used as inductors in osteogenic, adipogenic and chondrogenic differentiation protocols. Collas et al. [14] refer on the epigenetic basis of both MSC and embryonic stem cell differentiation potential. The capability for gene expression, controlled by epigenetic modifications of DNA and chromatin are increasingly studied in stem cell populations as they seem to define stages of pluripotency.
As indicated previously, mainly in vitro systems have been used to describe MSC functions. Accordingly, Quinn and Flake [12] critically review the rather few studies demonstrating in vivo differentiation and integration of MSC after transplantation. Whereas prenatal models, like blastocyst injection, have alluded to a more permissive environment for engraftment, postnatal models demonstrate major discrepancies between systemic injections and site-specific administration. While the former has been rather disappointing by means of numbers of engrafting and functional integrating MSC, sitespecific administration has often led to the repair of at least various connective tissue defects.

Having mainly focused on the subpopulation of MSC, which however represents a still heterogeneous mixture of pluri- to unipotent cells, the article by Ratajczak et al. [15] leads over to enumerating the several populations having been identified within the bone marrow, including hematopoietic stem cells, endothelial progenitor cells and of course MSC [15]. But they also refer to populations with enhanced potential that express pluripotency markers known from embryonic stem cells, for example multipotent adult progenitor cells (MAPC) or very small embryonic-like (VSEL) stem cells. The authors finally discuss the important question whether any of these cells really resides within the bone marrow.

In summary, the recent advances in stem cell research in general, but in MSC research in particular, have led to a better but not yet completely satisfying state of knowledge. It is regarded worthwhile extending research on basic MSC biology which however should be transferable to standardize and harmonize MSC for clinical purposes. Accordingly, a close interaction between clinical and basic science and a common interest in elucidating MSC, but also other stem cell basics will hopefully provide a basis for a better characterization and selection of highly potent MSC. Safety should be considered of highest importance for developing MSC-based therapies, and safety can only be achieved with consistently increasing knowledge of the fundamental basics.

\section{References}

1 Dominici M, Le Blanc K, Mueller I, Slaper-Cortenbach I, Marini F, Krause D, Deans R, Keating A, Prockop D, Horwitz E: Minimal criteria for defining multipotent mesenchymal stromal cells. The international society for cellular therapy position statement. Cytotherapy 2006;8:315-317.

2 Dexter TM: Stromal cell associated haemopoiesis. J Cell Physiol Suppl 1982;1:87-94.

$\checkmark 3$ Phinney DG, Prockop DJ: Concise review: Mesenchymal stem/multipotent stromal cells: The state of transdifferentiation and modes of tissue repair current views. Stem Cells 2007;25:2896-2902.

4 Caplan AI, Dennis JE: Mesenchymal stem cells as trophic mediators. J Cell Biochem 2006;98: 1076-1084.

$\checkmark 5$ Le Blanc K, Ringden O: Immunomodulation by mesenchymal stem cells and clinical experience. J Intern Med 2007;262:509-525.
6 Schäfer R, Northof H: Characteristics of mesenchymal stem cells - new stars in regenerative medicine or unrecognized old fellows in autologous regeneration? Transfus Med Hemother 2008;35(3): 154-159.

7 Bourin P, Gadelorge M, Peyrafitte J-A, Fleury-Cappellesso S, Gomez M, Rage C, Sensebe L: Mesenchymal progenitor cells: tissue origin, isolation and culture. Transfus Med Hemother 2008;35(3): 160-167.

8 Rojewski MT, Weber BM, Schrezenmeier H: Phenotypic characterization of mesenchymal stem cells from various tissues. Transfus Med Hemother 2008; 35(3):168-184.

9 Wagner W, Saffrich R, Ho AD: The stromal activity of mesenchymal stem cells. Transfus Med Hemother 2008;35(3):185-193.

10 Bifari F, Lisi V, Mimiola E, Pasini A, Krampera M: Immune modulation by mesenchymal stem cells. Transfus Med Hemother 2008;35(3):194-204.
11 Gimble JM, Guilak F, Nuttall ME, Sathishkumar S, Vidal M, Bunnell BA: In vitro differentiation potential of mesenchymal stem cells. Transfus Med Hemother 2008;35(3):228-238.

12 Quinn C, Flake AW: In vivo differentiation potential of mesenchymal stem cells: prenatal and postnatal model systems. Transfus Med Hemother 2008;35(3):239-247.

13 Frith J, Genever P: Transcriptional control of mesenchymal stem cell differentiation. Transfus Med Hemother 2008;35(3):216-227.

14 Collas P, Noer A, Sorensen AL: Epigenetic basis for the differentiation potential of mesenchymal and embryonic stem cells. Transfus Med Hemother 2008;35(3):205-215.

15 Ratajczak MZ, Zuba-Surma EK, Wojakowski W, Ratajczak J, Kucia M: Bone marrow - home of versatile stem cells. Transfus Med Hemother 2008;35 (3):248-259. 